\title{
Mechanistic Study on the Bis(p-sulfonatophenyl)phenylphosphine Synthesis of Monometallic Pt Hollow Nanoboxes Using Ag*-Pt Core- Shell Nanocubes as Sacrificial Templates
}

\author{
Yen-Nee Tan, ${ }^{\mathrm{b}}$ Jun Yang, ${ }^{\mathrm{a}}$ Jim Yang Lee, ${ }^{*}{ }^{\mathrm{a}, \mathrm{b}}$ and Daniel I.C. Wang ${ }^{\mathrm{b}, \mathrm{c}}$ \\ ${ }^{a}$ Department of Chemical and Biomolecular Engineering, National University of \\ Singapore. 10 Kent Ridge Crescent, Singapore 119260. Fax: 656779 1936; Tel: 656516 \\ 2899; E-mail: cheleejy@ nus.edu.sg \\ ${ }^{\mathrm{b}}$ Singapore-MIT Alliance, 4 Engineering Drive 3, National University of Singapore, \\ Singapore 117576 \\ ${ }^{c}$ Department of Chemical Engineering, Massachusetts Institute of Technology \\ Room 16-429, 77 Massachusetts Ave, Cambridge MA 02139 USA
}




\section{Supporting Information}

\section{S1: BSPP Assisted Dissolution of AgCl Nanoparticles}

Samples were drawn from $\mathrm{AgCl}$ nanoparticles recovered by centrifugation from the reaction mixture before chemical reduction, which were washed copiously with deionised water to remove the unbound ions. Evidence for the dissolution of $\mathrm{AgCl}$ nanoparticles was provided by changes in the color of the solution and the disappearance of the nanoparticles in TEM images after the BSPP or saturated $\mathrm{NaCl}$ treatment.

(a)

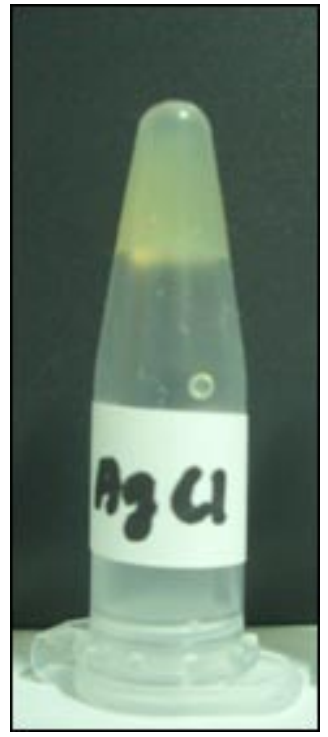

(b)

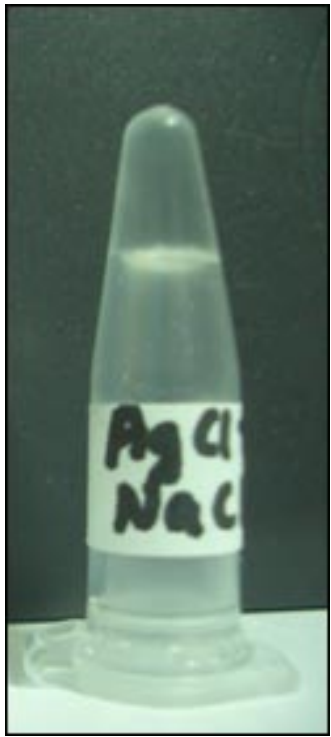

(c)

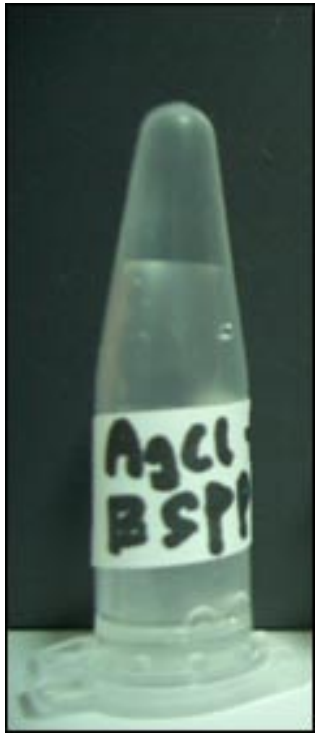

Figure S1. Digital photos of (a) $\mathrm{AgCl}$ nanoparticle solution; and the solubilzation of $\mathrm{AgCl}$ nanoparticles upon addition of (b) saturated $\mathrm{NaCl}$ and (c) $\mathrm{BSPP}$ to the $\mathrm{AgCl}$ solution in (a). 


\section{S2: Confirmation of BSPP-Ag Complex formation}

Two samples were prepared by mixing a $\mathrm{AgNO}_{3}$ solution with a $\mathrm{NaCl}$ solution. Solution (a) contained only the mixture of $\mathrm{AgNO}_{3}$ and $\mathrm{NaCl}$, while solution (b) contained dissolved $\mathrm{BSPP}, \mathrm{AgNO}_{3}$ and $\mathrm{NaCl}$. $\mathrm{AgCl}$ nanoparticles were readily precipitated in solution (a) while no precipitation occurred in solution (b). This shows that complexation of BSPP with $\mathrm{Ag}^{+}$had effectively shifted the $\mathrm{AgCl}$ equilibrium towards dissolution.

(a)

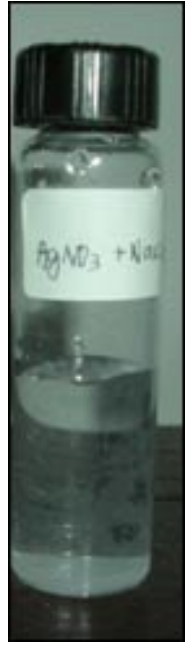

(b)

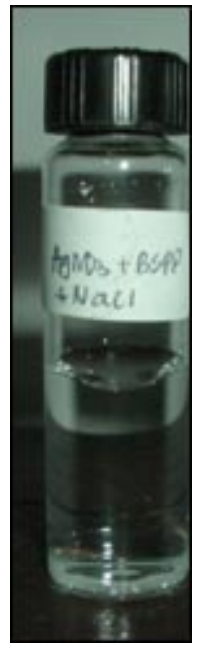

Figure S2. Digital photos of mixtures containing (a) $\mathrm{AgNO}_{3}$ and $\mathrm{NaCl}$ and (b) $\mathrm{AgNO}_{3}$, $\mathrm{NaCl}$ and dissolved BSPP. 